\title{
Induced Pluripotent Stem Cell Lines Derived from Equine Fibroblasts
}

\author{
Kristina Nagy • Hoon-Ki Sung • Puzheng Zhang • Simon Laflamme • Patrick Vincent • \\ Siamak Agha-Mohammadi • Knut Woltjen • Claudio Monetti • \\ Iacovos Prodromos Michael • Lawrence Charles Smith • Andras Nagy
}

Published online: 24 February 2011

(C) The Author(s) 2011. This article is published with open access at Springerlink.com

\begin{abstract}
The domesticated horse represents substantial value for the related sports and recreational fields, and holds enormous potential as a model for a range of medical conditions commonly found in humans. Most notable of these are injuries to muscles, tendons, ligaments and joints. Induced pluripotent stem (iPS) cells have sparked tremendous hopes
\end{abstract}

Kristina Nagy: Conception and design, collection and assembly of data, data analysis and interpretation, manuscript writing

Hoon-Ki Sung: Collection and assembly of data, data analysis and interpretation

Puzheng Zang: Collection and assembly of data

Simon Laflamme: Collection and assembly of data

Patrick Vincent: Collection and assembly of data

Siamak Agha-Mohammadi: Provision of study material

Knut Woltjen: Provision of study material, conception and design

Claudio Monetti: Collection and assembly of data

Iacovos P. Michael: Collection and assembly of data

Lawrence C. Smith: Conception and design, provision of study

materials, data analysis and interpretation

Andras Nagy: Conception and design, data analysis and interpretation, final approval of manuscript

This work was supported by funds from the Canadian Stem Cell Network and the Canadian Research Chair in Stem Cells and Regeneration (AN) and the Canadian Research Chair in Animal Cloning and Stem Cells (LCS).

K. Nagy $\cdot$ H.-K. Sung $\cdot$ P. Zhang $\cdot$ K. Woltjen $\cdot$ C. Monetti $\cdot$

I. P. Michael $\cdot$ A. Nagy

Samuel Lunenfeld Research Institute, Mount Sinai Hospital,

Toronto, ON, Canada

S. Laflamme $\cdot$ P. Vincent $\cdot$ L. C. Smith $(\bowtie)$

Faculty of Veterinary Medicine, Centre de Recherche en

Reproduction Animale, Universite de Montreal,

Saint-Hyacinthe, Canada

e-mail:smithl@medvet.umontreal.ca

S. Agha-Mohammadi

Hillman Cancer Center, University of Pittsburgh Medical Center, Pittsburgh, PA 15261, USA for future regenerative therapies of conditions that today are not possible to cure. Equine iPS (EiPS) cells, in addition to bringing promises to the veterinary field, open up the opportunity to utilize horses for the validation of stem cell based therapies before moving into the human clinical setting. In this study, we report the generation of iPS cells from equine

\author{
A. Nagy $(\bowtie)$ \\ Department of Molecular Genetics, University of Toronto, \\ Toronto, Canada \\ e-mail: nagy@mshri.on.ca
}

Present Address:

K. Woltjen

Center for iPS Cell Research and Application (CiRA), Institute for Integrated Cell-Material Sciences (iCeMS), Kyoto University, Kyoto 606-8507, Japan 
fibroblasts using a piggyBac (PB) transposon-based method to deliver transgenes containing the reprogramming factors Oct4, Sox2, Klf4 and c-Myc, expressed in a temporally regulated fashion. The established iPS cell lines express hallmark pluripotency markers, display a stable karyotype even during long-term culture, and readily form complex teratomas containing all three embryonic germ layer derived tissues upon in vivo grafting into immunocompromised mice. Our EiPS cell lines hold the promise to enable the development of a whole new range of stem cell-based regenerative therapies in veterinary medicine, as well as aid the development of preclinical models for human applications. EiPS cell could also potentially be used to revive recently extinct or currently threatened equine species.

Keywords Induced pluripotent stem cells · iPS cells . Equine $\cdot$ Horse $\cdot$ Transposon - Tetracycline inducible . Non-viral $\cdot$ PiggyBac

\section{Introduction}

The establishment of mouse Embryonic Stem (ES) cells 30 years ago $[1,2]$ gave rise to an unprecedented expansion of the field of genetics based on the mouse as a model organism. Soon after, a large effort was launched to find the equivalent tool for other species as well. Discouraging as it may be, the establishment of ES cells from many other species has been tried and has failed for a long time. Only very recently, as we have gained a deeper understanding of signaling pathways crucial for maintaining embryonic cells in the pluripotent state [3], has the establishment of rat embryonic stem cells been successful $[4,5]$. Attempts to derive equine ES cells face formidable challenges due to the difficulty and costs associated with recovering the needed embryos. Earlier, however, two groups have reported the derivation of equine ES-like cell lines [6, 7], but these did not give rise to teratomas when transplanted into immune deficient mice. Hence their genuine pluripotent state is uncertain.

When the first successful derivation attempts of iPS cells were reported in the mouse $[8,9]$ and later in the human $[10$, 11], huge hopes arose for using these as superior alternatives for ES cells in future cell-based therapies. Indeed, this field is developing with a tremendous speed, and to date, iPS cells have successfully been established from several species such as rhesus monkey [12], rat [13], dog [14], pig [15, 16], marmoset [17] and rabbit [18]. The derivation of equine iPS (EiPS) cells has however not yet been reported.

The horse represents a tremendous value both as a sport and companion animal, and as an excellent model for certain human diseases. Bone fracture is a common and very serious injury in these large animals as they respond poorly to standard treatment used successfully in other species (including human). Casting and long-term immobilization, for example, is usually not possible or is accompanied by high risks of devastating secondary complications. Damaged cartilage, tendons and ligaments have a poor capacity to heal not only in horses but in other species as well. Recently, therapeutic approaches using autologous mesenchymal stem cell (MSC) grafting have been developed in the horse $[19,20]$ and reviewed by Frisbie [21]. While equine MSCs have been shown to improve the early healing response in articular cartilage lesions, they do not enhance long-term tissue repair over that of controls [22]. The use of pluripotent stem cells may circumvent such issues, as these cells cannot only be expanded to large quantities in vitro, but they can also be directed to differentiate into a vast array of specific progenitor or terminally differentiated cell types. Evidence of success using such cell-based therapies has recently been reported [23]. These authors engineered tendon grafts that effectively repaired injured Achilles tendons in mice. Their study provided clear evidence that pluripotent stem cells indeed can be used to treat orthopedic injuries successfully.

Initially, somatic cell reprogramming to a pluripotent state was achieved by transducing mouse fibroblasts through viral vectors carrying the Oct4, Klf4, cMyc and Sox 2 transgenes [9]. To avoid the apparent drawbacks of the viral system, alternative methods have recently been developed [24-27]. We have previously reported the use of the piggyBac (PB) transposons - as a transgene deliverycombined with tetracycline inducible system for efficient generation of mouse and human iPS cells [28].

In the present study, we report the establishment of equine iPS (EiPS) cell lines using the same combination of $\mathrm{PB}$ transposon and tetracycline inducible system, with the addition of a green fluorescent protein (GFP) marker. Our EiPS cell lines exhibit properties of pluripotency such as alkaline phosphatase activity, expression of Oct4, Nanog, SSEA1, SSEA4, TRA-1-60 and TRA-1-81, and they display morphology similar to iPS cells from other species. Furthermore, these lines can be propagated for extended time periods in vitro without loosing a normal diploid karyotype, and most importantly, they are capable of forming teratomas containing tissues representing all three germ layers; ectodermal, mesodermal and endodermal.

\section{Materials and Methods}

Preparation of Equine Fetal Fibroblast (EFF) Cells

Viable fetuses were obtained from a local slaughterhouse in compliance with the guidelines of the Canadian 
Council on Animal Care. The age of the fetuses was calculated by measuring crown-rump length and estimated to day 55 of gestation. Skin was dissected from equine fetuses and exposed to a $5 \mathrm{mg} / \mathrm{ml}$ collagenase I (Sigma \#C0130) solution in DMEM for $3 \mathrm{~h}$ at $37^{\circ} \mathrm{C}$. After dissociation, cells from each fetus were washed twice in fresh DMEM with $10 \%$ FBS, plated, cultured, and passaged once to obtain first passage equine fetal fibroblasts (EFFs). EFF stocks were frozen in DMEM with $10 \%$ FBS and $10 \%$ DMSO.

\section{Cell Culture}

Equine fetal fibroblasts were maintained in DMEM High Glucose (Invitrogen 11960-044) supplemented with $2 \mathrm{mM}$ GlutaMax $^{\text {TM }}$ (Invitrogen \# 35050), $0.1 \mathrm{mM}$ Non-essential amino acids (Invitrogen \# 11140), $0.1 \mathrm{mM}$ Betamercaptoethanol (Sigma M7522), $1 \mathrm{mM}$ Sodium Pyruvate (Invitrogen \#11360-070), $50 \mathrm{U} / \mathrm{ml}$ Penicillin/Streptomycin (Invitrogen \#15070) and 15\% fetal bovine serum (HyClone). Culture media for EiPS cells was the same as above, supplemented with $1000 \mathrm{U} / \mathrm{ml}$ leukemia inhibitory factor (LIF; Millipore \#ESGRO), $10 \mathrm{ng} / \mathrm{ml} \mathrm{bFGF} \mathrm{(Peprotech} \mathrm{\# 100-18B),} 1.5 \mu \mathrm{g} / \mathrm{ml}$ Doxycycline (Sigma \#D9891) (unless otherwise stated), $3 \mu \mathrm{M}$ GSK inhibitor (StemGent \#CHIR99021), 0.5 M MEK inhibitor (StemGent \#PD0325901), $2.5 \mu \mathrm{M}$ TGF inhibitor (StemGent \#A83-01) and Thiazovivin (StemGent \#Thiazovivin). From day 8 of the reprogramming process until the third passage (app day 30) the media was also supplemented with $25 \mu \mathrm{M}$ ALK receptor inhibitor (StemGent \#SB431542).

\section{Plasmid Construction}

The PB-TET-MKOS and PB-CAG-rtTA plasmids were constructed as previously described [28]. The transposase expression vector (pCyL43 PBase) [28] was obtained from Sanger (http://www.sanger.ac.uk/technology/clonerequests). PB-GFP was generated by replacing the NheI (polished) HindIII excised PGK-neo cassette from PB-neo [28] with a SalI (polished) HindIII fragment composed of the CAG promoter, GFP coding region, and rabbit beta globin polyadenylation signal.

\section{Transfection}

EFFs were transfected with the Neon electroporation device (Invitrogen) according to the manufacturers instruction, using preset program 14. For each electroporation in $10 \mu \mathrm{l}$ tips, $5 \times 10^{4}$ cells were used with a total of $1 \mu \mathrm{g}$ mixed DNA. The DNA mixture consisted of equal weight ratios of PB-TET-MKOS, PB-CAG-rtTA, PB-CAG-GFP and pCyL43. Cells were seeded at a density of $5 \times 10^{4}$ cells per well of a $9.6 \mathrm{~cm}^{2}$ surface area.

\section{Establishment of EiPS Cell Lines}

Colonies were picked mechanically on day $17-22$ posttransfection without any passaging of the cells prior to picking. The first passages used mechanical dissociation. From the third passage onward, the lines were propagated enzymatically with TrypLE Select (Invitrogen \#12563), cultured on mitomycin inactivated feeder layers consisting of $50 \%$ mouse embryonic fibroblasts isolated from E14.5 embryos with standard procedure (MEFs) and 50\% EFF. The cells were passaged every 23 days at a 1:4 ratio. At the appropriate level of expansion, the cells were cryopreserved in $90 \%$ FBS and $10 \%$ DMSO.

\section{AP and LacZ Staining}

Alkaline Phosphatase staining was performed on cells grown in 4-well tissue culture plates. The cells were washed once with DPBS and stained with Vector laboratories AP staining kit according to the manufacturers instructions. For LacZ staining, adherent cultures were washed once in DPBS and fixed for $10 \mathrm{~min}$ in $0.02 \%$ glutaraldehyde (Sigma \#D6257) in DPBS. The cultures were then washed 3 times in DPBS and stained overnight at $37^{\circ} \mathrm{C}$ in the dark in the following staining solution: $1 \mathrm{mg} /$ $\mathrm{mL}$ X-Gal in N,N-dimethylformamide, $5 \mathrm{mM} \mathrm{K}_{4} \mathrm{Fe}(\mathrm{CN})_{6}$ and $5 \mathrm{mM} \mathrm{K}_{3} \mathrm{Fe}(\mathrm{CN})_{6}$ (all components from Sigma).

\section{Southern Blotting}

To detect the integration site and copy number of the reprogramming transgenes, ten micrograms of genomic DNA was digested with BamHI overnight, resolved by gel electrophoresis, and transferred to Hybond $\mathrm{N}+$ membrane (GE Healthcare \#RPN303B). A neo probe PCR fragment prepared with Amersham Rediprime ${ }^{\mathrm{TM}}$ II DNA Labeling System (GE Healthcare \# RPN1633) was used for hybridization. The detection of the rtTA transgene was done the same way, except that the genomic DNA was cut with HindIII and an rtTA probe was used.

\section{Karyotyping}

EiPS cells were grown on $60 \mathrm{~mm}$ diameter tissue culture dishes in the described medium and on fibroblast feeder layers until semi-confluent. Following enzymatic dissociation, the cells were re-suspended in culture media, centrifuged, washed once in DPBS, centrifuged again and re-suspended in $1 \mathrm{ml}$ DPBS in a $15 \mathrm{ml}$ conical tube. $10 \mathrm{ml}$ of $0.8 \%$ sodium citrate in $\mathrm{H}_{2} \mathrm{O}$ was added slowly, the tube was inverted 3 times and incubated at $37^{\circ} \mathrm{C}$ for $30 \mathrm{~min}$. Fixative (1 $\mathrm{ml}$ of 1:3 acetic acid/methanol) was added and 
then the tube was inverted 3 times and centrifuged at $2000 \mathrm{rpm}$ for $2 \mathrm{~min}$. The supernatant was decanted leaving approximately $1 \mathrm{ml}$ behind, and the tube flicked to resuspend the cells. Fixative was added slowly while gently vortexing the tube, and the centrifugation and decanting step was repeated. The cell suspension was moved to a $1.5 \mathrm{ml}$ micro-centrifuge tube and the fixation/washing step repeated two more times. Chromosome spreads were prepared by dropping $20 \mu \mathrm{l}$ cell suspension onto cold ultra-clean microscope slides placed in a warm and humid atmosphere. After 5 minutes drying at $56^{\circ} \mathrm{C}$, the slides were mounted with Vectashield mounting medium with DAPI (Vector lab \#1200).

\section{RT-PCR}

RNA was collected from cells grown in $60 \mathrm{~mm}$ tissue culture plates by brief enzymatic dissociation, resuspension with culture media, centrifugation, and resuspension in Trizol (Invitrogen \#15596-026). RNA was extracted by isopropanol and purified with RNeasy Mini kit (Qiagen \#74104) following manufacturer's protocol. Reverse transcription was performed using Quanti Tect Reverse Transcription kit (Qiagen \#205313) with RT primers Mix in the kit. The reaction was performed at $42^{\circ}$ $\mathrm{C}$ for $60 \mathrm{~min}$. For all samples, a negative RT was used as a control, consisting of an RT reaction omitting the reverse transcriptase. In addition, polymerase chain reaction (PCR) omitting the cDNA was used as a negative control. The following RT-PCR primers were used to detect the expression of equine specific $\boldsymbol{\beta}$-actin (forward: ATGGTGGCAATGGGTCAGAAGGAC, reverse: CTCTTTGATGTCACGCACGATTTC, amplify 550 bp), Klf4 (forward: AGGCATCTCCCATTTATATTTA, reverse: CATGGGGAGAGCTCCTC, amplify 332 bp), Nanog (forward: TCCTCAATGACAGATTTCAGAGA, reverse: GAGCACCAGGTCTGACTGTT, amplify 323 bp), Oct4 (forward: GGGACCTCCTAGTGGGTCA, reverse: TGGCAAATTGCTCGAGGTCT, amplify 318 bp). None of these primer pairs amplify mouse transcrips.

\section{In Vitro Differentiation}

EiPS cells were seeded on non-adherent culture plates (Corning 3471) at a density of $0.5-1 \times 10^{5}$ cells per $9.6 \mathrm{~cm}^{2}$ culture area (3 $\mathrm{cm}$ dish) in medium consisting of DMEM High Glucose (Invitrogen 11960-044) supplemented with 2 mM GlutaMax ${ }^{\mathrm{TM}}$ (Invitrogen \#35050), $0.1 \mathrm{mM}$ Betamercaptoethanol (Sigma \#M7522), 50U/ml Penicillin/ Streptomycin (Invitrogen \#15070) and 5\% fetal bovine serum (HyClone). After 5 days in suspension, the embryoid bodies (EBs) were plated in 4-well plates coated with a 1:10 dilution of Matrigel (BD \#356234) in DPBS.
Immunocytochemistry

Cells grown in 4-well tissue culture plates or on coverslips were washed twice with DPBS (Invitrogen) and fixed with $3.7 \%$ paraformaldehyde solution for $10 \mathrm{~min}$. After three times washing with DPBS, the cells were permeabilized with $1 \%$ triton for $10 \mathrm{~min}$ and blocked $30 \mathrm{~min}$ with $5 \%$ normal goat serum (Jackson ImmunoResearch) in DPBS. The cells were then incubated overnight at $4^{\circ} \mathrm{C}$ with the following primary anti-mouse or anti-human antibodies; $(i)$ anti-Nanog (Reprocell \#RCAB0002P-F), (ii) anti-Oct4 (Santa Cruz \#sc-5279), (iii) anti-SSEA1 (Stemgent \#090005), (vi) anti-SSEA4 (Stemgent \#09-0006), (v) anti-TRA1-60 (Stemgent \#09-0009), (vi) anti-TRA-1-81 (Stemgent \#09-0011). After several washes in DPBS, cells were incubated for 45-120 min at room temperature with Cy3, Cy5, FITC or or Allexa 488 conjugated secondary antibodies (Jackson ImmunoResearch or Molecular Probes). For control experiments, the primary antibody was omitted or replaced by pre-immune serum, or we used an isotypematched control or purified rabbit IgG.

\section{Teratomas}

EiPS cells were harvested by TrypLE Select (Invitrogen \#2563) enzymatic dissociation, and $5 \times 10^{6}$ cells were resuspended in $100 \mu 1$ Matrigel diluted 1:3 with DMEM. The cell suspensions were injected subcutaneously into the dorsal flanks of NOD/SCID (NOD.CB17-Prkdc-scid/J, Jackson Laboratories) mice. The mice were housed a specific pathogen free (SPF) condition and the care of the animals was in accordance with institutional guidelines (Toronto Centre for Phenogenomics, Toronto, Ontario, Canada). The mice were given doxycycline by feeding them doxycycline supplemented food pellets $(200 \mathrm{mg} / \mathrm{kg}$, Bioserv cat \# S3888) for 4 weeks to aid the survival and engraftment of the iPS cells. Three to four months after injection, teratomas were dissected, fixed overnight in $10 \%$ buffered formalin phosphate and embedded in paraffin. Sections were stained with hematoxylin and eosin.

Imaging

Bright field and fluorescence images of live cells and EBs were acquired on a Leica DMIL inverted microscope equipped with a QImaging Retica 12-bit color digital camera and OpenLab software. Chromosome spreads were imaged on a Leica DMR inverted microscope equipped with a 100x oil immersion lens, a Leica DC300F digital camera and QWin software. Immunofluorescent signals were visualized and digital images were obtained by using a Zeiss LSM 510 confocal microscope equipped with two photon, argon and helium-neon lasers (Zeiss). Confocal 
imaging was also performed using an Olympus FV1000 laser-scanning confocal microscope. The acquisition of images was done with Fluoview 1000 software and the time of acquisition of fluorescent samples was 10 microseconds with a resolution of 1024 X 1024 pixels. Kalman filter was applied to remove noise background. The acquired images were then exported as TIFF files and processed using Olympus Fluoview software FV10-ASW (Olympus Canada, Markham, ON, Canada).

\section{Results}

Generation and in Vitro Characterization of EiPS Cell Lines

We set out to derive iPS cells from equine fetal fibroblasts using our previously established PB transposon-based system to deliver the reprogramming factors $[28,29]$. The main feature of this approach lies in a vector in which cMyc, Klf4, Oct4 and Sox2 (MKOS) are linked by 2A peptide sequences (i.e. MKOS) [29]. In order to gain temporal control over the reprogramming process, the four factors are placed under the transcriptional control of the tetO2 promoter (PB-TET-MKOS) (Fig. 1a1). An IRESBgeo cassette following MKOS renders the cells both neomycin resistant as well as LacZ positive upon Dox treatment. The tetracycline reverse transactivator (PB-CAGrtTA) (Fig. 1a2), a vector containing a constitutive GFP expression cassette (PB-CAG-GFP) (Fig. 1a3), as well as a circular transient PB transposase (pCyL43 PBase) [28] expression vector (Fig. 1a4) were all co-electroporated with the PB-TET-MKOS plasmid. We electroporated passage 3 fibroblasts (Fig. 1b1) and plated them at a density of approximately $1.5 \times 10^{5}$ cells per $30 \mathrm{~mm}$ culture dish. By comparing the number of live cells in transfected versus non-transfected cells on the day following electroporation, we estimated the survival rate to be $75 \%$. We estimated the transfection efficiency to $46 \%$ (data not shown) from the ratio of GFP positive cells.

The reprogramming process was induced by adding Dox to the culture medium 2 days after transfection. At this time, the media was also supplemented with LIF and bFGF, a GSK-inhibitor (CHIR99021), TGFbeta-inhibitor (A8301), MEK-inhibitor (PD0325901) cocktail [3] and Thiazovivin [30]. On day 8 post-transfection, the ALK receptor inhibitor SB431542 was added to the media as well [30]. This compound was maintained in the media only from day 8 to 30 , while the other inhibitors and Dox were added to the media at all times. On day 14 post-transfection, focal areas of increased proliferation could be observed. Within these regions, the cells took on a more rounded and densely growing morphology. Three to 4 days later, many of these areas developed into clearly defined colonies (Fig. 1b2), which were then picked on day 17-22 post-transfection. After an initial three mechanical passages, the cells could be enzymatically dissociated with TrypLE Select and split at a rate of 1:4 every 2-3 days. The cells survived cryopreservation and thawing, and we were able to expand them to large quantities.

Initially, we kept the cultures growing on a feeder layer consisting of MEFs. When we placed the EiPS cells on a EFFs feeder layer, the growth rate slowed down but the colonies took on a denser and more well defined appearance (data not shown). By mixing MEFs and EFFs at a 1:1 ratio, we obtained what seemed to be the most advantageous conditions for EiPS cells. On this feeder composition, the colonies grow at the same rate as when grown on MEFs, and they maintain dense colony morphology.

Using three independent fetal fibroblast sources, we successfully established a total of 25 EiPS cell lines. The reprogramming efficiency was estimated to $0.028 \%$ by dividing the number of transfected $\left(\mathrm{GFP}^{+}\right)$cells surviving electroporation by the number of colonies found on each plate (data not shown). This rate is similar to those obtained when reprogramming human and mouse fibroblasts with the same transfection system [28].

\section{In Vitro Characterization of EiPS Cell Lines}

The EiPS colonies exhibited morphology similar to human iPS cells with monolayer organization, well-defined edges (Fig. 1b3) and a high nuclear to cytoplasm ratio (Fig. 1b4). As expected, the lines were positive for LacZ expression while the media was supplemented with Dox, proving that the transgenes were active (Fig. 1b5). Alkaline phosphatase activity could also clearly be detected (Fig. 1b6).

Co-electroporation of the PB-CAG-GFP vector resulted in approximately half of the resulting EiPS cell lines being $\mathrm{GFP}^{+}$ (data not shown). For further analysis, we focused on those lines that strongly expressed this fluorescent marker (Fig. 1c).

Southern blotting using probes specific both for the combined reprogramming factors (Fig. 2a1) as well as for the rtTA transgene (Fig. 2a2) revealed that most lines had a relatively low number of MKOS integrations and a higher number of integrations of the PB-CAG-rtTA transposon.

We then examined the chromosome number integrity in 13 EiPS cell lines. After preparing mitotic chromosome spreads (Fig. 2b), we counted the chromosomes from 3050 cells for each line. Some lines exhibited a low ratio of euploid cells, but the majority possessed an euploid count in at least $80 \%$ of the cells (Fig. 2c). We followed the chromosome count stability of three EiPS cell lines over increased passage numbers and could confirm that the euploid rate was only slightly decreased at least up until passage number 26 (data not shown). 
Fig. 1 EiPS cell derivation. (a). The reprogramming vector set. 1. PB-TET-MKOS vector containing the tetracycline inducible reprogramming factors (c-Myc, Klf4, Oct4 and Sox2) and an IRES linked Bgeo. 2. PB-CAGrtTA vector providing expression of the tetracycline reverse transactivator. 3. PB-CAG-GFP vector allowing expression of the Enhanced Green Fluorescent Protein. 4. pCyL43-PBase piggyBac transposase expression vector. (b). Morphology of equine cells in vitro. 1. Equine fetal fibroblasts. 2. Primary EiPS colony. 3. Colonies during expansion using enzymatic passaging. 4. High magnification photograph of EiPS cell showing high nucleus to cytoplasm ratio (arrow). 5. LacZ staining reveals transgene expression. 6. EiPS cells are alkaline phosphatase positive. (c). GFP expression in EiPS colonies. Scale bars $50 \mu \mathrm{m}$

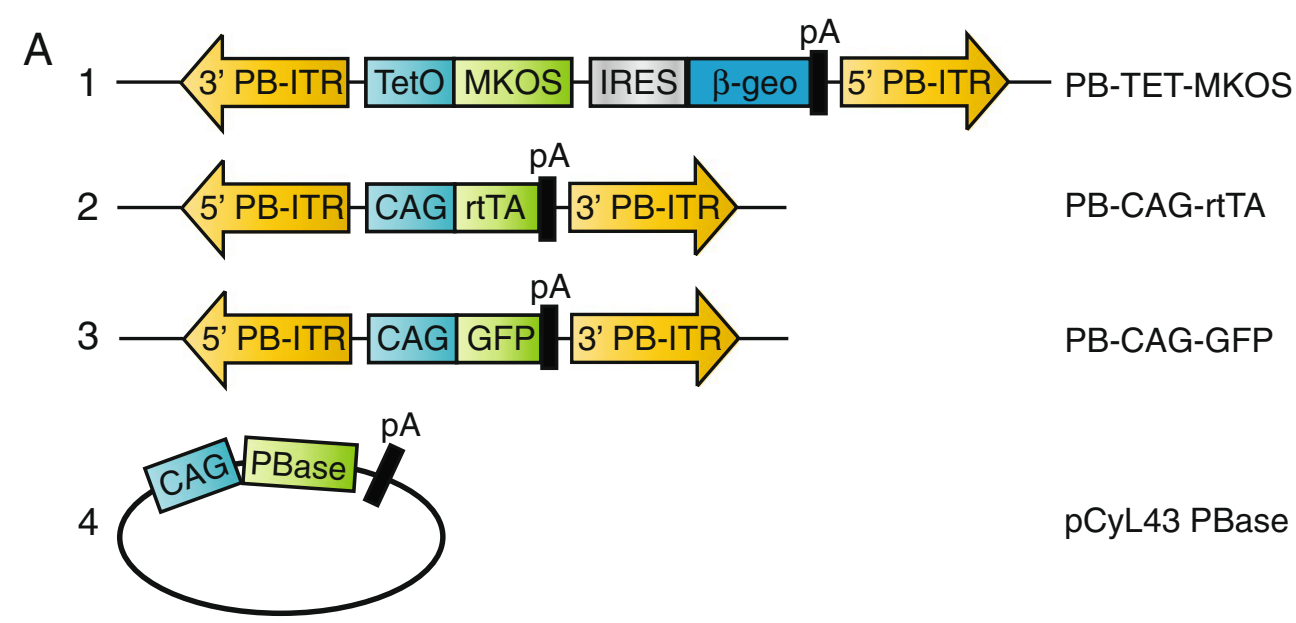

B
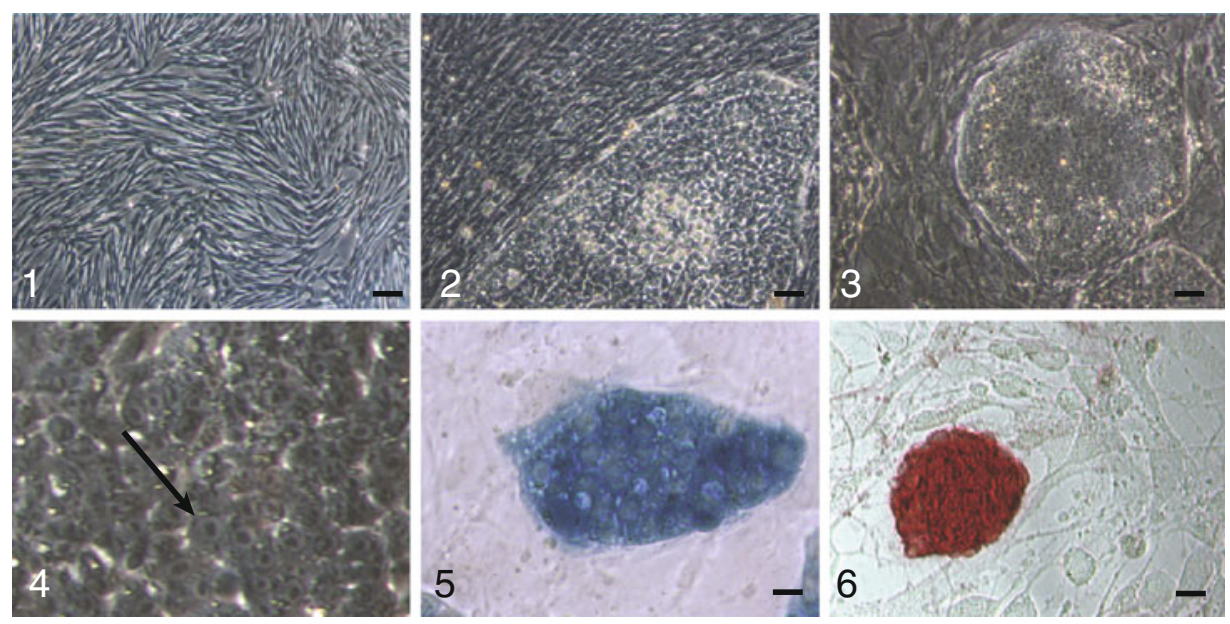

C
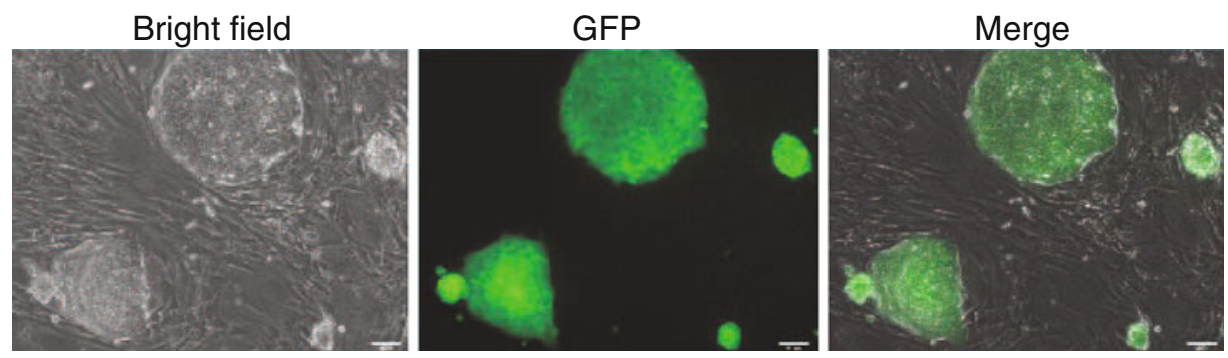

Using immunocytochemistry assays, we could detect expression of the key pluripotency markers Nanog, SSEA1, SSEA4, Tra-1-60 and Tra-1-81 (Fig. 3a). In addition, RT-PCR using horse-specific primers, confirmed a robust expression of equine endogenous Oct4, Nanog and Klf4 (Fig. 3b).

Next, we created embryoid bodies (EBs) from EiPS cells to assess their developmental potential in vitro. After 4 days of suspension culture in media lacking LIF, FGF, inhibitors and Dox, the cells formed EBs (Fig. 4a) that already on day 4 developed fluid filled cysts (Fig 4a arrow). This cyst formation is unusually early and may indicate an accelerated differentiation of EiPS cells compared to mouse and human pluripotent stem cells. This notion was further supported by the observation that the horse EBs tended not to aggregate to each other at higher densities and they did not attach to standard tissue culture plastic or a variety of substrates such as Laminin, Collagen or Fibronectin. Matrigel-coated tissue culture surface, however, allowed day 6 EBs to attach and expand into a variety of cell types. Unfortunately, due to the lack of suitable antibodies raised against equine cells [31], we were not able to perform immunocytochemical analyses of these differentiated cultures. To compensate for this shortcoming, we focused on characterizing the pluripotency of our EiPS cell lines using in vivo assays. 
Fig. 2 Genomic and cytogenetic properties of EiPS cell lines. (a). Southern blotting analysis to detect integration of the 1 . PBTET-MKOS and 2. PB-CAGrtTA transgenes. Number of integration sites are indicated at the bottom of each membrane. (b). DAPI stained mitotic chromosome spread. (c). Ratio of cells with normal (euploid) chromosome counts among EiPS cell lines
A
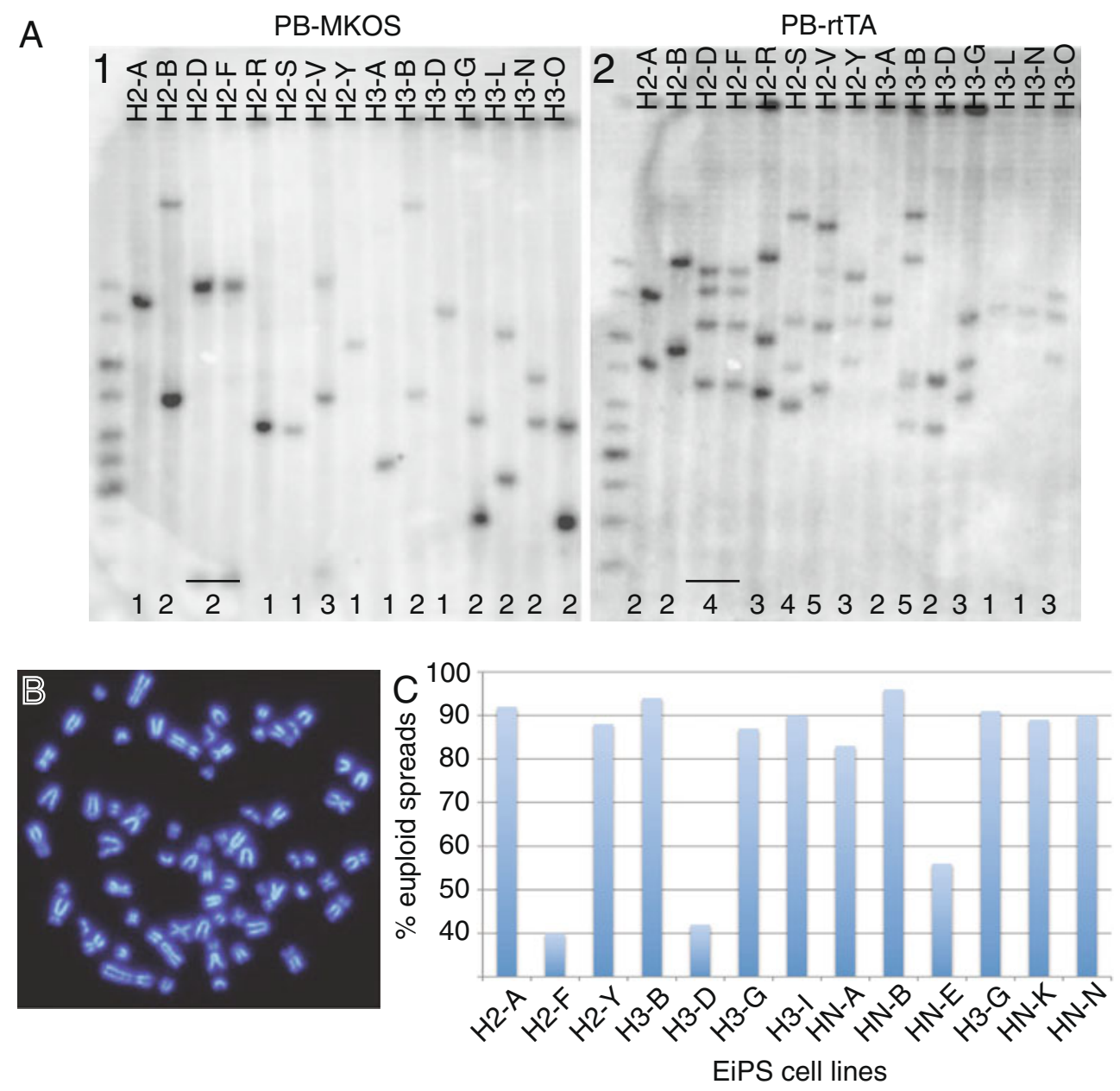

\section{In Vivo Characterization of EiPS Cell Lines}

We continued our in vivo characteization by implanting our EiPS cells into immunocompromised mice. The presence of GFP expression was strong also after differentiation into large teratomas (Fig 4b). Four months after implantation, we were able to harvest teratomas that contained cell types derived from all three embryonic germ layers, indicating that our EiPS cell lines are truly pluripotent cells (Fig. 4c). Most importantly, these tumors did not show any signs of malignancy. They were very well defined and did not produce any metastatic tumors (data not shown).

\section{Discussion}

We report here the successful establishment of equine iPS (EiPS) cells. Building on our previous experience, we chose the PB transposon system to deliver the tetracycline inducible reprogramming transgenes c-Myc, Klf4, Oct4 and Sox2 into equine fetal fibroblasts (EFFs). Based on Southern blotting analysis, we could determine that the number of integration sites for each transgene was relatively low, in most cases between 1 and 4 (Fig 2a). The higher copy number for the rtTA transposon may be due to a higher molecular ratio upon transfection as equal weights of plasmid were used despite the smaller size of PB-CAG-rtTA (7016 bp) as compared to PB-TET-MKOS (14216 bp). As evident by the Southern blotting analysis for PB-TET-MKOS and PB-CAG-rtTA transposons, the lines $\mathrm{H} 2-\mathrm{D}$ and $\mathrm{H} 2-\mathrm{F}$ are genetically identical (Fig. 2a) and therefore most likely the result of the same reprogramming event.

The characterization of these lines posed a significant challenge mainly due to the lack of antibodies that are specific for equine pluripotency markers [31] limiting us to use those that are raised against mouse or human and by chance cross-react with the corresponding equine factors.

We attempted to assess the capacity of EiPS cells to differentiate in vitro by producing EBs that subsequently were allowed to adhere and grow out under in vitro 
Fig. 3 In vitro characterization of the pluripotent state of EiPS cells. (a). Nanog, SSEA1, SSEA4, TRA-1-60 and TRA-1-81 detected by fluorescent immunohistochemistry. Yellow scale bar $10 \mu \mathrm{m}$, white scalebar $20 \mu \mathrm{m}$. (b). Detection of gene expression of key pluripotency markers using RT-PCR primers specific for equine endogenous genes
A

DAPI $A b$
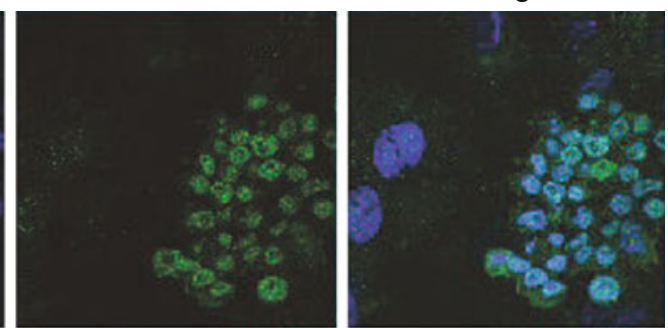

SSEA1
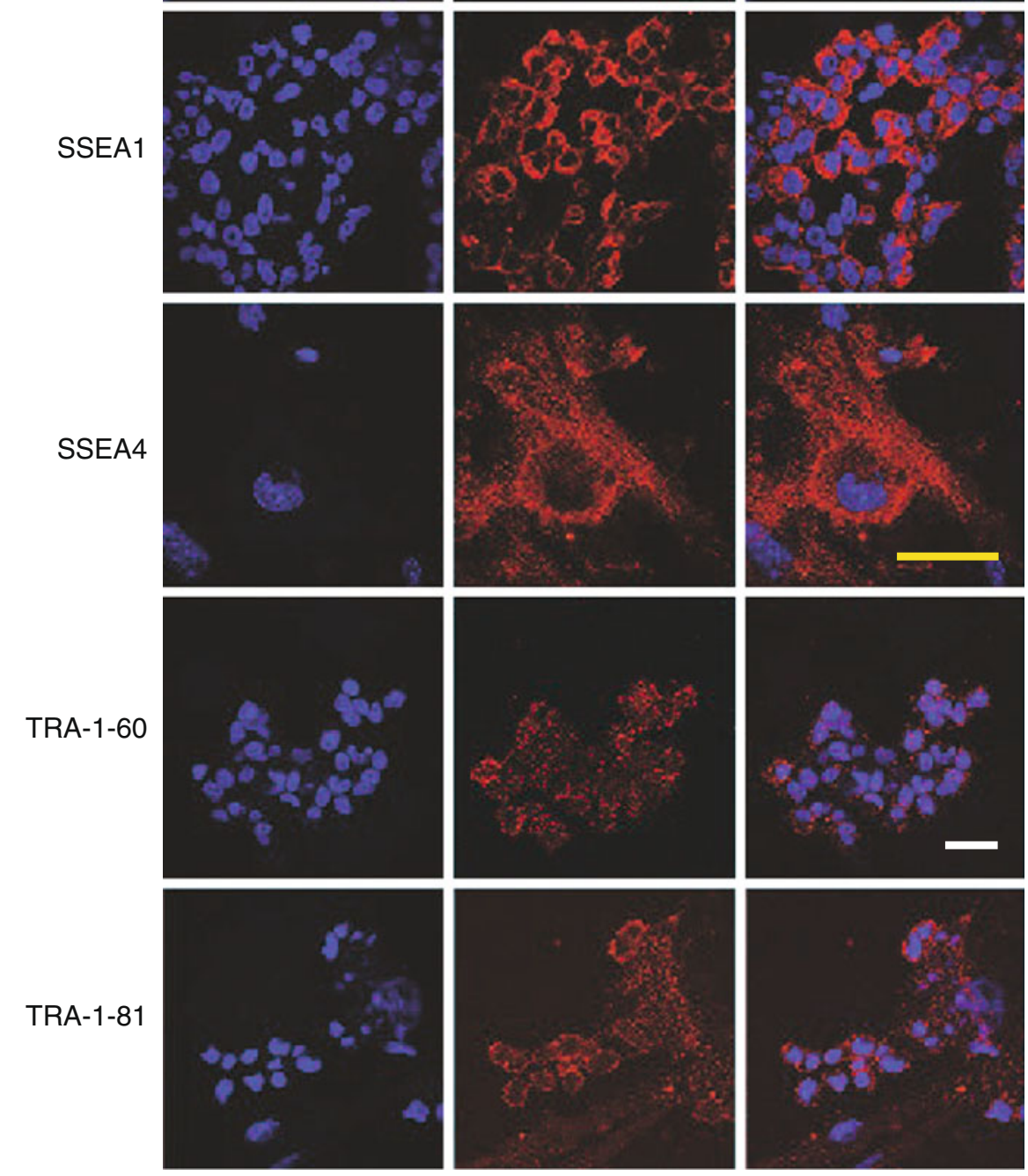

B
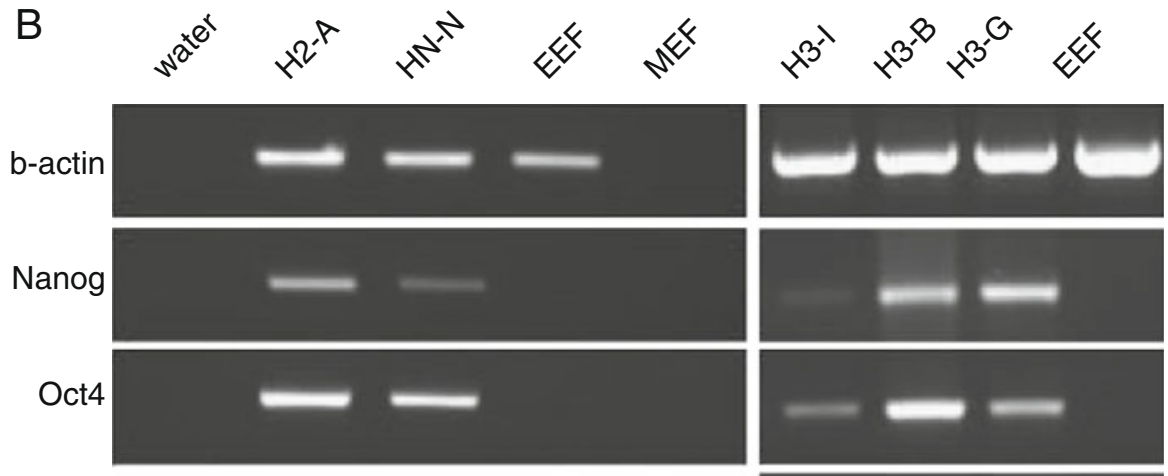

Klf4 
Fig. 4 Characterization of the differentiation potential of EiPS cells. (a). Embryoid bodies

(EBs) that become cystic (white arrow). (b). Teratoma in situ on the hind limb of a mouse (arrow pointing at the tumor), showing strong GFP expression. (c). Teratoma assay; $\mathbf{1}-\mathbf{3}$, derivatives of the embryonic endoderm: $\mathbf{1}, \mathbf{2}$. Intestine-like structures, black arrows $(1,2)$, and respiratory epithelium, black arrow (3). 4$\mathbf{6}$, derivatives of the embryonic mesoderm: Bone, black arrows (4), cartilage (5), and muscle, black arrows (6). 7-9, derivatives of the embryonic ectoderm: Neuroepithelium, black arrow (7), glycogeneted epithelium, black arrow (8), and skin epithelium (black arrows) and keratin (yellow arrow) (9)
A

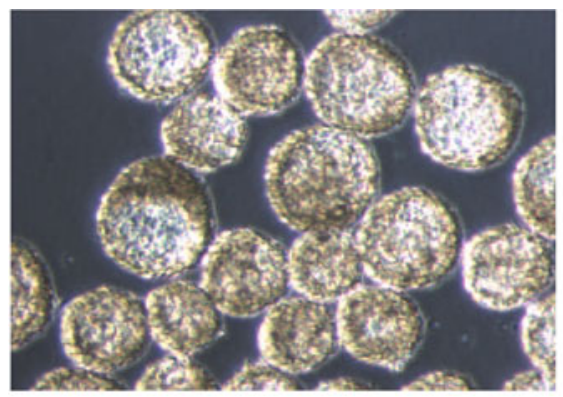

B

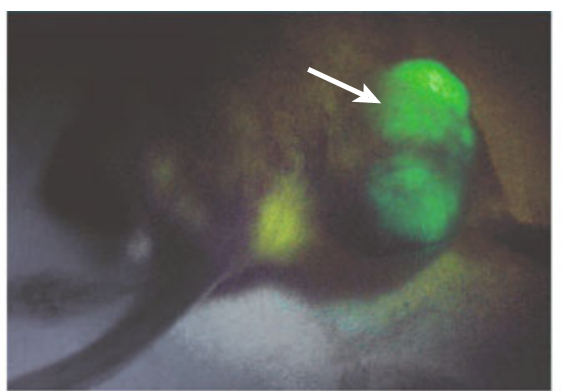

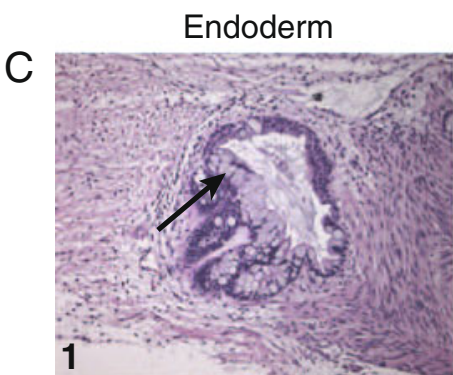
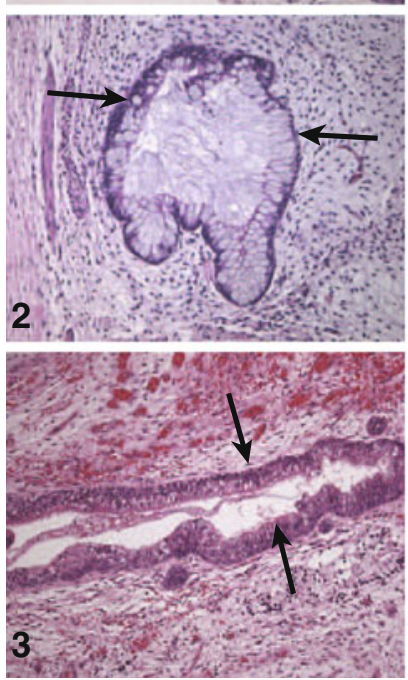
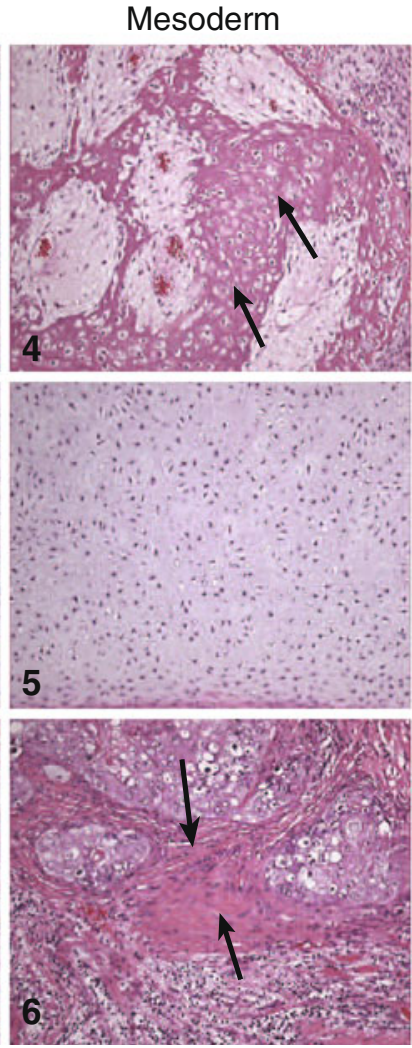
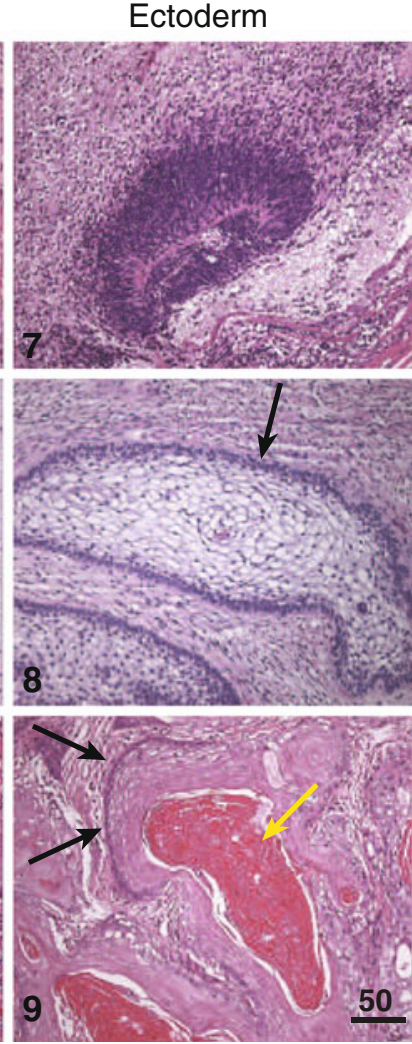

differentiation conditions. Suitable antibodies to detect specific cell lineages in the equine differentiating cells are unfortunately not available, and this limitation restricted our ability to characterize the cells by immunocytochemical assays. The extremely long time it would take to examine the developmental potential of EiPS cells in vivo through aggregation with equine embryos places this experiment beyond the scope of this report. With these challenges is mind, we decided to perform the next most stringent in vivo test of pluripotency we did have at hand; formation of teratomas in immunocompromised mice. Our initial attempts resulted in very slow growth of the teratomas; not until 6 months after initial implantation of EiPS cells did we observe tumor formation. We attributed this finding to the notion - supported by the unusually rapid in vitro differentiation of EiPS cells into cystic embryoid bodiesof an accelerated differentiation of these cells in permissive environments. To counteract this putative property of the cells, we fed the recipient mice with doxycycline for 4 weeks to inhibit with the premature final differentiation of the engrafted iPS cells. We then removed Dox from the animals diet to let the grafted cells differentiate and form teratomas. Fully differentiated teratomas with derivatives of all three germ layers then readily formed within 2 months after the Dox treatment was stopped.

Efforts have long been ongoing to establish pluripotent stem cells from horses. Access to such cell lines would not only be of great value for veterinary medicine, but would also open up possibilities to use the horse as an excellent pre-clinical model for future human stem cell-based 
therapies. We report on the successful derivation of iPS cells from equine fibroblasts. Our EiPS cell lines display a pluripotent phenotype based on expression of key markers, alkaline phosphatase activity, and the formation of complex teratomas representing all three embryonic germ layers.

Acknowledgements We thankfully acknowledge Malgosia Kownacka for preparation of cell culture reagents and Esther Lau for technical assistance with cell culture. We are also grateful to Dr. Peter Tonge for his invaluable intellectual input. This work was supported by a grant to AN from the Canadian Stem Cell Network and to LCS from the Canadian Research Chair in Animal Cloning and Stem Cells.

Conflict of Interest The authors declare no potential conflicts of interest.

Open Access This article is distributed under the terms of the Creative Commons Attribution Noncommercial License which permits any noncommercial use, distribution, and reproduction in any medium, provided the original author(s) and source are credited.

\section{References}

1. Evans, M. J., \& Kaufman, M. H. (1981). Establishment in culture of pluripotential cells from mouse embryos. Nature, 292, 154-156.

2. Martin, G. R. (1981). Isolation of a pluripotent cell line from early mouse embryos cultured in medium conditioned by teratocarcinoma stem cells. Proceedings of the National Academy of Sciences of the United States of America, 78, 7634-7638.

3. Ying, Q. L., Wray, J., Nichols, J., et al. (2008). The ground state of embryonic stem cell self-renewal. Nature, 453, 519-523.

4. Buehr, M., Meek, S., Blair, K., et al. (2008). Capture of authentic embryonic stem cells from rat blastocysts. Cell, 135, 1287-1298.

5. Demers, S. P., \& Smith, L. C. (2010). Derivation, culture, and in vivo developmental capacity of embryonic cell lines from rat blastocysts. Methods Mol Biol, 597, 179-188.

6. Li, X., Zhou, S. G., Imreh, M. P., Ahrlund-Richter, L., \& Allen, W. R. (2006). Horse embryonic stem cell lines from the proliferation of inner cell mass cells. Stem Cells and Development, 15, 523-531.

7. Saito, S., Ugai, H., Sawai, K., et al. (2002). Isolation of embryonic stem-like cells from equine blastocysts and their differentiation in vitro. FEBS Letters, 531, 389-396.

8. Okita, K., Ichisaka, T., \& Yamanaka, S. (2007). Generation of germline-competent induced pluripotent stem cells. Nature, 448, 313-317.

9. Takahashi, K., \& Yamanaka, S. (2006). Induction of pluripotent stem cells from mouse embryonic and adult fibroblast cultures by defined factors. Cell, 126, 663-676.

10. Takahashi, K., Tanabe, K., Ohnuki, M., et al. (2007). Induction of pluripotent stem cells from adult human fibroblasts by defined factors. Cell, 131, 861-872.

11. Yu, J., Vodyanik, M. A., Smuga-Otto, K., et al. (2007). Induced pluripotent stem cell lines derived from human somatic cells. Science, 318, 1917-1920.

12. Liu, H., Zhu, F., Yong, J., et al. (2008). Generation of induced pluripotent stem cells from adult rhesus monkey fibroblasts. Cell Stem Cell, 3, 587-590.
13. Liao, J., Cui, C., Chen, S., et al. (2009). Generation of induced pluripotent stem cell lines from adult rat cells. Cell Stem Cell, 4, 11-15.

14. Shimada, H., Nakada, A., Hashimoto, Y., Shigeno, K., Shionoya, Y., \& Nakamura, T. (2010). Generation of canine induced pluripotent stem cells by retroviral transduction and chemical inhibitors. Molecular Reproduction and Development, 77, 2.

15. Esteban, M. A., Xu, J., Yang, J., et al. (2009). Generation of induced pluripotent stem cell lines from Tibetan miniature pig. The Journal of Biological Chemistry, 284, 17634-17640.

16. Ezashi, T., Telugu, B. P., Alexenko, A. P., Sachdev, S., Sinha, S., \& Roberts, R. M. (2009). Derivation of induced pluripotent stem cells from pig somatic cells. Proceedings of the National Academy of Sciences of the United States of America, 106, 10993-10998.

17. Wu, Y., Zhang, Y., Mishra, A., Tardif, S. D., \& Hornsby, P. J. (2010). Generation of induced pluripotent stem cells from newborn marmoset skin fibroblasts. Stem Cell Res, 4, 180-188.

18. Honda, A., Hirose, M., Hatori, M., et al. (2010). Generation of induced pluripotent stem cells in rabbits: potential experimental models for human regenerative medicine. The Journal of Biological Chemistry, 285, 31362-31369.

19. Nathan, S., De Das, S., Thambyah, A., Fen, C., Goh, J., \& Lee, E. H. (2003). Cell-based therapy in the repair of osteochondral defects: a novel use for adipose tissue. Tissue Engineering, 9, 733-744.

20. Taylor, S. E., Smith, R. K., \& Clegg, P. D. (2007). Mesenchymal stem cell therapy in equine musculoskeletal disease: scientific fact or clinical fiction? Equine Veterinary Journal, 39, 172-180.

21. Frisbie, D. D., \& Smith, R. K. (2010). Clinical update on the use of mesenchymal stem cells in equine orthopaedics. Equine Veterinary Journal, 42, 86-89.

22. Wilke, M. M., Nydam, D. V., \& Nixon, A. J. (2007). Enhanced early chondrogenesis in articular defects following arthroscopic mesenchymal stem cell implantation in an equine model. Journal of Orthopaedic Research, 25, 913-925.

23. Cohen, S., Leshansky, L., Zussman, E., et al. (2010). Repair of full-thickness tendon injury using connective tissue progenitors efficiently derived from human embryonic stem cells and fetal tissues. Tissue Engineering. Part A, 16, 3119-3137.

24. Kim, D., Kim, C. H., Moon, J. I., et al. (2009). Generation of human induced pluripotent stem cells by direct delivery of reprogramming proteins. Cell Stem Cell, 4, 472-476.

25. Okita, K., Nakagawa, M., Hyenjong, H., Ichisaka, T., \& Yamanaka, S. (2008). Generation of mouse induced pluripotent stem cells without viral vectors. Science, 322, 949-953.

26. Stadtfeld, M., Nagaya, M., Utikal, J., Weir, G., \& Hochedlinger, K. (2008). Induced pluripotent stem cells generated without viral integration. Science, 322, 945-949.

27. Zhou, H., Wu, S., Joo, J. Y., et al. (2009). Generation of induced pluripotent stem cells using recombinant proteins. Cell Stem Cell, 4, 381-384.

28. Wang, W., Lin, C., Lu, D., et al. (2008). Chromosomal transposition of PiggyBac in mouse embryonic stem cells. Proceedings of the National Academy of Sciences of the United States of America, 105, 9290-9295.

29. Kaji, K., Norrby, K., Paca, A., Mileikovsky, M., Mohseni, P., \& Woltjen, K. (2009). Virus-free induction of pluripotency and subsequent excision of reprogramming factors. Nature, 458, 771-775.

30. Lin, T., Ambasudhan, R., Yuan, X., et al. (2009). A chemical platform for improved induction of human iPSCs. Natural Methods, 6, 805-808.

31. Paris, D. B., \& Stout, T. A. (2010). Equine embryos and embryonic stem cells: defining reliable markers of pluripotency. Theriogenology, 74, 516-524. 